\title{
Simulating multiple inheritance and generics in Java
}

\author{
Krishnaprasad Thirunarayan ${ }^{\mathrm{a}, *}$, Günter Kniesel $^{\mathrm{b}}$, Haripriyan Hampapuram ${ }^{\mathrm{c}}$ \\ ${ }^{a}$ Department of Computer Science and Engineering, Wright State University, Dayton, OH-45435, USA \\ ${ }^{\mathrm{b}}$ Computer Science Department III, University of Bonn, D-53117 Bonn, Germany \\ ${ }^{\mathrm{c}}$ Intrinsa Corporation, Mountain View, CA-94041, USA
}

Received 4 August 1999; accepted 27 June 2000

\begin{abstract}
This paper presents Java language from an object-oriented software construction perspective. It explains the implications of banning generics and multiple inheritance of classes, and explores the patterns and the idioms used by the Java designers and programmers to redeem their benefits. The paper also discusses an alternative to multiple inheritance, as incorporated in Lava, which extends Java with constructs for type-safe automatic forwarding. (c) 2001 Elsevier Science Ltd. All rights reserved.
\end{abstract}

Keywords: Java language; Object-oriented programming; Design patterns; Multiple inheritance; Generics; Delegation

\section{Introduction}

Design patterns are a description of communicating objects and classes that are customized to solve a general design problem in a particular context [1-3]. Design patterns consolidate design experience that can potentially be reused in tackling a variety of problems, and in organizing and analyzing potential solutions to the problem. Many of these patterns improve software reuse and facilitate code evolution.

Idioms, on the other hand, are low-level "patterns" that describe how to solve implementationspecific problems in a programming language. Idioms demonstrate competent use of programming language features [2].

Java does not support generics and multiple inheritance of classes, thereby simplifying the language and its implementation. This paper discusses the implications of Java's lack of generics and

\footnotetext{
${ }^{*}$ Corresponding author. Tel.: +1-937-775-5109; fax: +1-937-775-5133.

E-mail addresses: tkprasad@cs.wright.edu (K. Thirunarayan),gk@cs.uni-bonn.de (G. Kniesel), hhampa@microsoft.com (H. Hampapuram).
} 
multiple inheritance of classes on software construction, and presents techniques to mitigate its effect.

Section 2 reviews problems associated with support for multiple inheritance in existing objectoriented languages. Section 3 analyzes idiomatic approximations to multiple inheritance in Java using only single inheritance of classes and multiple inheritance of interfaces. It also rationalizes certain aspects of Java in terms of design patterns. Section 4 discusses possible language support for multiple inheritance. It shows that asking for multiple inheritance in Java is not a good idea given that more powerful, dynamic alternatives are available. In particular, support for delegation would be preferrable to adding multiple inheritance.

Section 5 analyzes idiomatic approximations to generics using reference types and reflection, and discusses their limitations vis-a-vis an explicit support for generics. Section 6 concludes the paper.

\section{Background: multiple inheritance}

Object-oriented languages such as Eiffel, $\mathrm{C}++$, etc., support multiple inheritance of classes, while Smalltalk, Oberon, Modula-3, Ada-95, etc., support only single inheritance of classes [4]. Furthermore, Eiffel, $\mathrm{C}++$, Ada-95, etc., support generics/templates to promote reuse. In contrast, Java supports neither multiple inheritance of classes nor generics, explicitly. This simplifies the language and its implementation but "burdens" the programmers and the Java API designers.

There is no consensus among researchers on the semantics of multiple inheritance in the presence of method overriding and potential conflicts due to multiple definitions. (Bertrand Meyer [5] provides a lucid account of the key issues and insights for incorporating multiple inheritance in an object-oriented language. Similar issues have also been studied by artificial intelligence and object-oriented databases communities [6].) The programming language designers have taken at least three different approaches, exemplified by the design of $\mathrm{C}++$, Eiffel and Java.

\section{1. $C++$}

$\mathrm{C}++$ views multiple inheritance as replicated single inheritance. That is, a child class inherits every field/method of every parent class, unless the field/method is overridden by the child. In case more than one parent defines the same field/method, the child uses the scope-resolution operator to disambiguate the reference. The problem of repeated inheritance [5] of a field/method arises when the parents share a common ancestor. In $\mathrm{C}++$, the fields in a child instance due to a common ancestor are either all shared (virtual) or all duplicated. This approach has two serious drawbacks in practice: (1) the "all or none" granularity in choosing to share or to duplicate fields of a common ancestor, and (2) the place where this choice is made [4,7]. Another consequence of the $\mathrm{C}++$ decision is that several natural implementations, such as the code for the Tree data structure using leftmost-child-right-sibling form given below, are deemed illegal.

class Cell : public Link $\{\ldots\}$;

class Tree: public Cell, public Link $\{\ldots\}$; 
However, the corrected version, given in Chapter 13 of [8], requires unreasonable amount of code duplication that an OOPL is fighting hard to minimize.

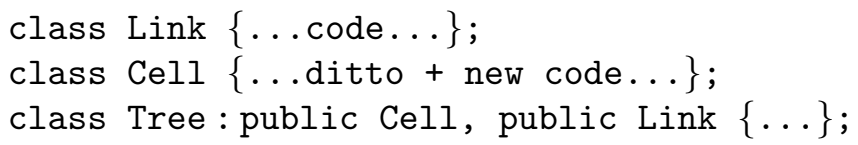

To paraphrase S. Tucker Taft's posting to comp.lang.ada: The $\mathrm{C}++$ mechanism for multiple inheritance has the worst of both the worlds: a language made more complex by the addition of linguistic multiple inheritance, and an application made more difficult by the lack of appropriate building blocks for solving the problem the "right" way.

\subsection{Eiffel}

In contrast, Eiffel provides a rich set of primitives to program-in an application-specific strategy for multiple inheritance [5]. In particular, it supports:

redefinition: to override a (potentially inheritable) field/method definition.

renaming: to remove name clashes making available multiple definitions under different names.

undefinition: to "join" names and to make unavailable certain definitions.

selection: to pick the code that must be run on a subclass instance referenced by an entity of a "repeated" ancestor type using dynamic binding in case of an ambiguity.

Whereas the flexibility and the expressiveness that Eiffel's design of multiple inheritance endows is widely recognized, it is an open debate whether it commensurates with the additional complexity introduced in the language and its implementation.

Furthermore, Eiffel's select mechanism exhibits a subtle semantic problem of its own. Because it always selects the same code, irrespective of the context of the method invocation, it reinstalls the problem that method renaming was intended to solve: the selected method is executed even in contexts where a method with the same signature but different semantics is expected. A detailed discussion is beyond the scope of this paper and can be found in [9].

\subsection{Java}

The Java designers have chosen to eliminate multiple inheritance of classes. Instead, the programmer is now expected to realize multiple inheritance by "simulating" it in the language. This decision is motivated by the problems sketched above and the additional desire for binary compatibility: Recall that in Java, the computation of numeric offsets for instance fields in memory is done by the run-time system. This permits updating a class with new instance fields or methods without affecting existing code. In other words, it is possible to run an existing bytecode file for a subclass after updating and recompiling the class, but without recompiling the subclass. This property would be lost if multiple inheritance were supported because multiple inheritance conflicts could suddenly arise during class loading.

Note also that, on older processors, single inheritance of classes had performance advantages over multiple inheritance because the former simplified the layout of instance fields and enabled generation of efficient code for dynamic dispatching of methods [10]. However, modern pipelined processors execute the additional offset adjustment instruction during the delay time required for branching to 
the method's code [11]. So, surprisingly, multiple inheritance overheads are negligible even in the presence of polymorphic variables.

We now explore the extent to which the reusability benefits of multiple inheritance and generics can be reinstated in Java through specific patterns and idioms.

\section{Approximating multiple inheritance in Java}

What does support for multiple inheritance accomplish for the following example, which is illegal in Java?

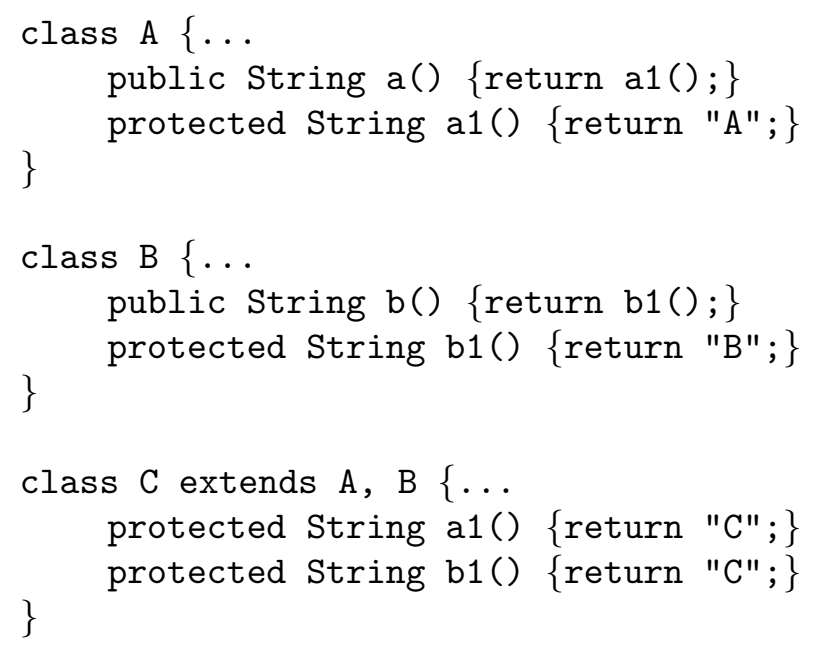

Code reuse: The fields defined in classes $\mathrm{A}$ and $\mathrm{B}$ are present in an instance of class $\mathrm{C}$, and a method defined in class A (resp. B) can potentially be run on an instance of class C.

Polymorphism: An instance of class C can potentially be used wherever an instance of class A (resp. B) is required.

Overriding: Inherited methods can be adapted to the needs of class $\mathrm{C}$ by overriding methods on which they rely. For instance, the definition of method $\mathrm{a} 1()$ and $\mathrm{b} 1()$ in $\mathrm{C}$ has the indirect effect that the inherited methods $\mathrm{a}()$ and $\mathrm{b}()$ now return " $\mathrm{C}$ ".

Modification: Any changes to classes A and B are propagated automatically to class C.

Often, multiple inheritance is (mis)used for just one of the above effects, for example, just for code reuse or for achieving multiple subtyping. It is, therefore, important to understand how each effect can be simulated in Java and, even more, to assess which effects are relevant to a particular design.

In the following, we will first present the general scheme for simulating multiple inheritance and identify the ingredients of the simulation that achieve each of the above effects. Then we will present different examples that illustrate how these ingredients can be used in various combinations. The examples will develop from cases that do not really require multiple inheritance (and are indeed better solved without), via cases where multiple inheritance would be appropriate (and can be approximated in Java), to cases where more dynamic techniques than multiple inheritance are required. We show that in the latter cases our simulation is preferable to multiple inheritance but it still cannot provide 
the full power that can be achieved by suitable language support. We conclude this section by contrasting our simulation with an extension of Java that supports multiple object-based inheritance.

\subsection{General scheme}

\subsubsection{Simple forwarding}

In a first attempt, the example above can be approximated in Java as follows (changes are underlined):

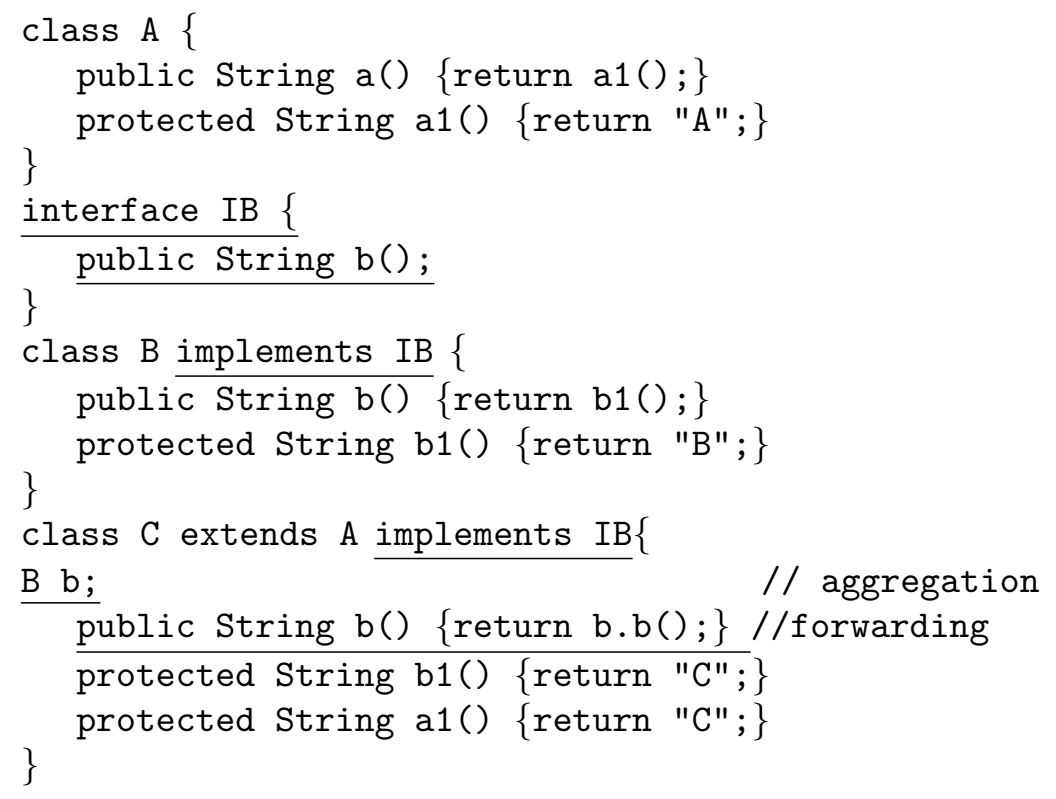

Class C inherits from class A and supports the same interface IB as class B. Furthermore, class C forwards every invocation of a method in interface IB to the corresponding field of type B.

The generalization of this translation to "class C extends A, B1, .., Bn $\{\ldots\}$ " is straightforward given that Java supports implementation of multiple interfaces. We call the "simulated superclasses" $\mathrm{B} i$ the parent classes of $\mathrm{C}$, in contrast to "genuine superclasses" like A. Similarly, $\mathrm{C}$ is called child class of each $\mathrm{Bi}$.

How well does this translation capture multiple inheritance?

Code reuse: Class $\mathrm{C}$ reuses the code for classes $\mathrm{A}$ and $\mathrm{B}$ but does require glue code to implement forwarding.

Polymorphism: An instance of class $\mathrm{C}$ can be used for an instance of class A and for an instance ${ }^{1}$ of interface IB but not for an instance of class B. The latter is because, in general, implementing the common interface IB does not imply that class $C$ supports all the methods in class $B$.

If interface IB contains all the public methods of class $B$, and IB is used instead of $B$ for type declarations throughout the program, then the ability to substitute $C$ instances for IB instances is sufficient. So, whether the translation supports polymorphism depends on anticipation of future extensions (the interface and the corresponding implements relation in the parent class must be

\footnotetext{
${ }^{1}$ By an instance of an interface we mean an object that is an instance of a class that implements the interface.
} 
declared), good documentation (of the intention that the interface should be used for typing instead of the parent class) and programmer discipline (you must adhere to the documentation).

Modification: Any changes to class A are automatically propagated to class C. Similarly, adding a new field to class B or changing a method body in class B is transparent to class C. However, the addition or deletion of a method from class $B$ or changes to a method signature in class $B$ requires changes to interface IB and to class $C$ (to install the glue code for forwarding).

Overriding: Overriding and the related adaptation of "inherited" code from aggregated objects is not possible. For instance, the definition of method b1() in $\mathrm{C}$ does not influence the result of the method $\mathrm{b}()$. An invocation of $\mathrm{a}()$ on an instance of class $\mathrm{C}$ returns " $\mathrm{C}$ " but an invocation of $\mathrm{b}()$ on an instance of class $C$ still returns "B".

Overriding is the core concept of inheritance [12,13]. Without overriding, existing class hierarchies would dramatically change their semantics and many essential concepts and techniques (for example, protected methods, abstract classes, template methods [1]) would not exist. Because it disables overriding, the above translation cannot be regarded as a satisfactory approximation of (either single or multiple) inheritance.

\subsubsection{Overriding via back-references}

Achieving the effect of overriding is also possible on the basis of aggregation and forwarding, provided that the parent classes are available for modification [14,15]. The essential idea is to give the parent object a back-reference to the object that initially forwarded the message. Messages otherwise sent to this by the parent are now sent via the back-reference, enabling execution of overriding methods from the child.

The back-reference can either be stored in the parent object or it can be passed dynamically as an additional argument of forwarded messages. The first approach is called the stored pointer model, the second one is called the passed pointer model [14]. A detailed discussion and usability evaluation of the passed pointer model and the stored pointer model can be found in [15]. Applying the passed pointer model to our example yields:

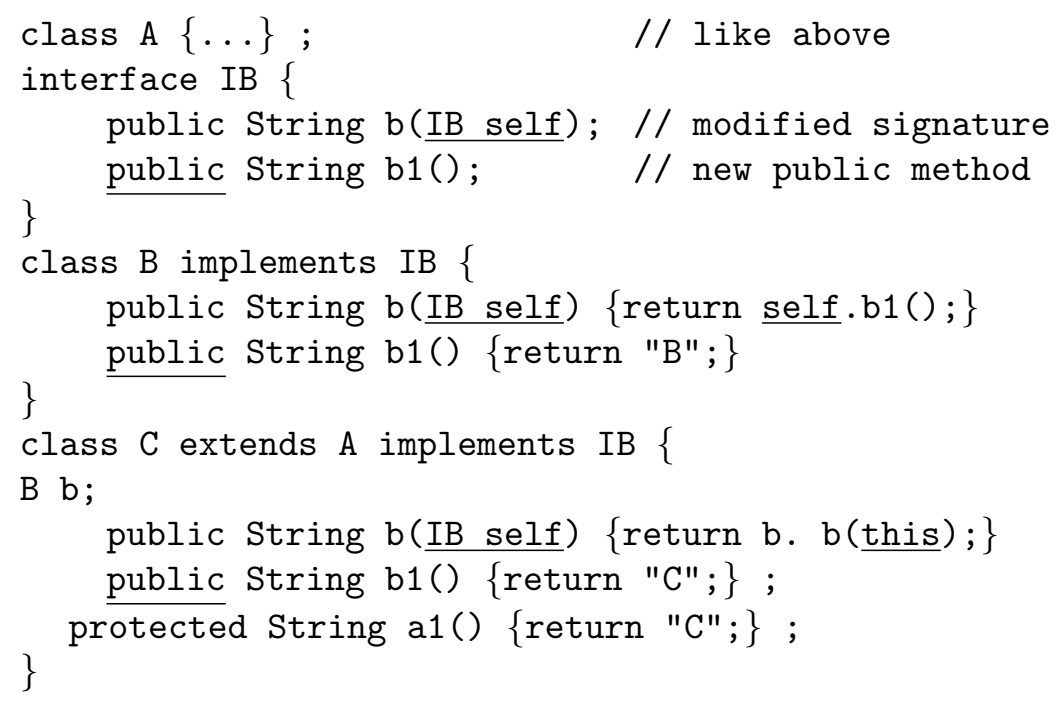


Now invocations of method $\mathrm{a}()$ and $\mathrm{b}()$ on an instance of class $\mathrm{C}$ both return the string "C". Note that we had to add the method b1() to interface IB and relax the visibility status of method b1() from protected to public, thus generally exposing details that should be known only to subclasses/child classes.

Meta-level techniques can also be used for simulating multiple inheritance and delegation [16]. However, these exhibit shortcomings with respect to type-checking and performance. Therefore, we have confined ourselves to non-reflective approaches.

\subsubsection{Conflicts prevent polymorphism}

If several (base) classes define methods that share the same signature, the translation sketched in this section permits just one method with that signature in the child class. This is a problem if the different base class methods have different semantics and child class instances are substitutable for base class instances: then a wrong method that happens to share the name and signature can be invoked. This problem is illustrated in [17, pp. 273-275] with the following example (adapted here to pseudo-Java syntax):

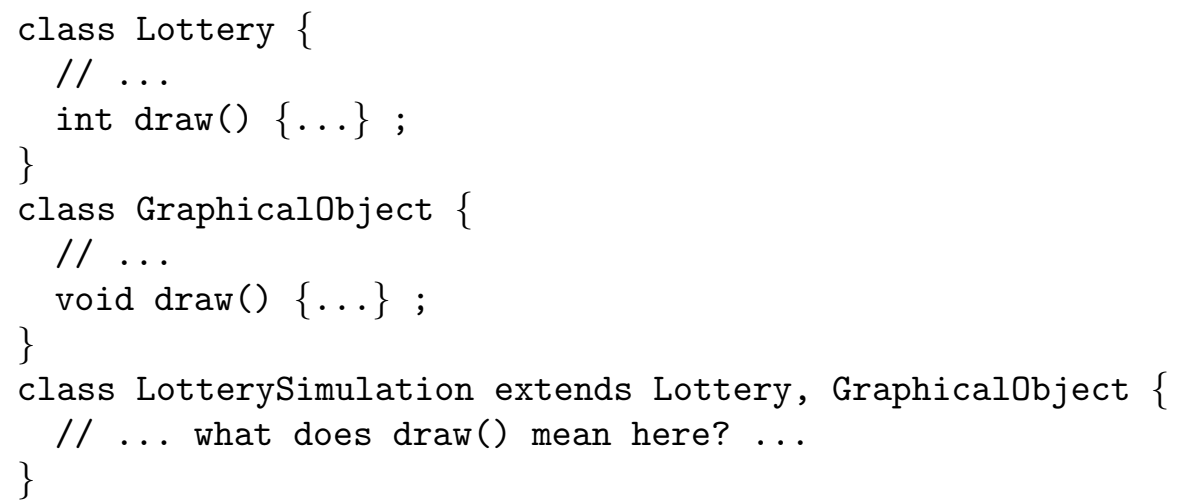

In such a case we need two methods in the LotterySimulation class, say drawLottery() and drawGraphical(). Without language support for multiple inheritance, however, dynamic binding does not know that it should select the drawLottery() method for an invocation of draw () on a variable of type Lottery and the drawGraphical() method for an invocation of draw() on a variable of type Graphicalobject.

Implementation of such context-dependent behavior without language support results in convoluted, impractical designs. This is problematic because it leaves us only with the choice to give up substitutability of a child class instance for a parent class instance (by not declaring the implements relation). As a consequence, child classes cannot override methods in the respective parent classes because the simulation of overriding depends on substitutability of child instances for parent class instances:

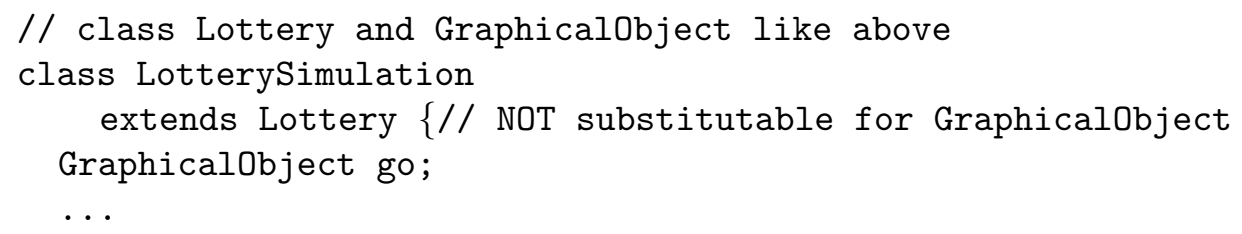




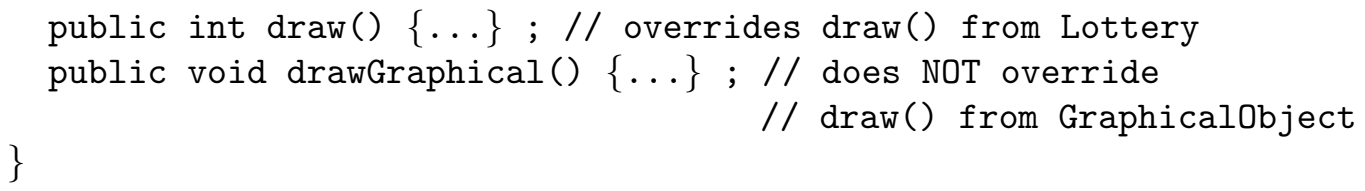

An example showing that much better results can be achieved with suitable language support is shown in Section 4.2.

\subsection{Illustrative examples}

To sum up, multiple inheritance can be simulated by joint use of

- forwarding as a means to achieve code reuse,

- interfaces as a means to achieve polymorphism, and

- back-references as a means to approximate overriding.

The applicability of the latter two techniques is inhibited, however, by the need to provide suitable "hooks" in parent classes. Furthermore, conflicting methods in parent classes prevent simulation of polymorphism, even if the parent classes provide the required hooks.

Whereas the above limitions of the available simulation techniques prevent a full simulation of multiple inheritance in cases where it would be useful, there are also other cases where a full simulation is not required and a design without multiple inheritance is preferable. We now present some practical applications of the above translation in Java, which illustrate different facets of both situations.

On the one hand, the examples review some widely used combinations of the three simulation techniques discussed above, showing that a rigid implementation of the presented translation is often neither appropriate nor possible. The right choice is driven by the needs of a particular application and constrained by the limitations of available built-in or third-party supplied classes that cannot be modified. On the other hand, they illustrate a number of specialized patterns derived from the above translation. Anticipating the need for customization, vendors can apply such patterns in designing utility classes and frameworks, to facilitate their use in multiple inheritance contexts.

\subsubsection{Code reuse without polymorphism}

One application of multiple inheritance is to provide an implementation of an abstraction, using a concrete class. This has been called "the marriage of convenience" in [5]. In $\mathrm{C}++$, private inheritance is used for a similar purpose. In both cases the inheriting class is not intended to be a subtype of the concrete class, it just reuses its code to implement the interface of the abstraction. In Java, this corresponds to a child class that forwards to the concrete class and inherits from an abstract class (or implements an interface) that represents the abstraction. An example would be an abstract class Stack ${ }^{2}$ that can be implemented using different underlying concrete data types, for example, an Array or a LinkedList.

\footnotetext{
${ }^{2}$ Note that this example does not reflect the current design of the Java APIs, where Stack is a class with one fixed implementation as a subclass of Vector. This includes all Vector operations in the interface of Stack, enabling to bring a Stack into an inconsistent state, for example, by directly calling the method add (atIndex, elem).
} 


\subsubsection{Code reuse and polymorphism}

In Graphical User Interfaces, "containers" such as windows, frames, panels, etc. hold primitive "components" such as buttons, check-boxes, text-fields, etc. In many applications, a group of such elements exhibits similar behavior as the individual element or is controlled similarly as the individual element. Java supports such recursive nestings by letting java.awt. Container inherit from java.awt.Component. In fact this is an instance of the well-known composite pattern [1] that appears in various other contexts such as in the implementation of:

- Command macros, where a sequence of commands is treated as a command.

- Composite figures, where a set of figures is treated as a figure.

- Nested menus, where a menu can be a menu-item.

The essence of the composite pattern is that composite elements behave at the same time like elements and like collections of elements. Thus they should be subtypes of both, Element and Collection. A composite element can ideally be defined using multiple inheritance as follows [5]:

$$
\begin{aligned}
& \text { class CompositeElement extends Collection, Element }\{\ldots\} \\
& \text { class CompositeElement extends Collection[Element], Element }\{\ldots\}
\end{aligned}
$$

In the latter case, Collection is a template/generic (see Section 5 for a discussion on generics in Java).

In Java, the composite pattern can be coded using single inheritance of classes by:

1. defining an interface to specify the methods required of each element (whether primitive or composite),

2. defining a class for the composite elements that (a) implements the interface, and (b) extends an existing collection class, and

3. requiring a client to manipulate the elements via the methods listed in the interface.

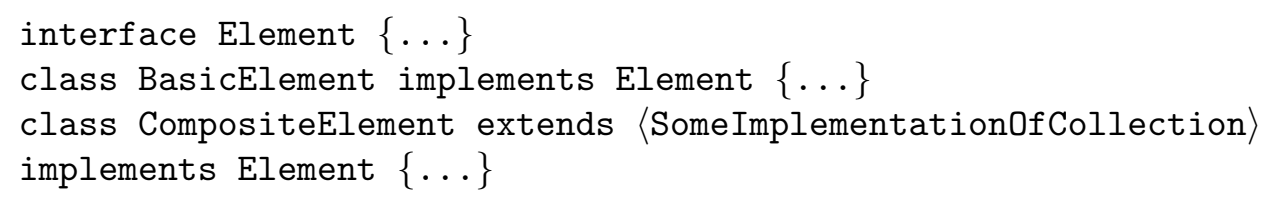

\subsubsection{Code reuse, selective overriding, no polymorphism}

Ideally, the well-known Clock applet class ought to multiply inherit from built-in classes java.applet.Applet and java.lang. Thread. That is,

Class Clock extends Applet, Thread $\{\ldots\}$;

This declaration, which is illegal in Java, enables the clock to run in a browser, and update itself periodically on a separate thread. To facilitate implementation of such classes by single inheritance, Java defines an interface

interface Runnable \{public void run();

and a Thread constructor of the form

Thread (Runnable r) $\{\ldots\}$. 
Now class Clock can be coded in Java as:

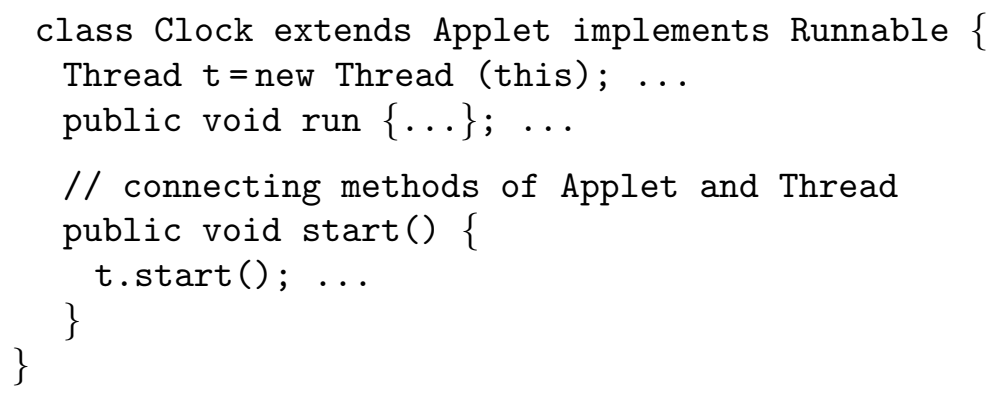

The class Thread exemplifies an implementation of overriding via stored back-references that are passed to the parent object in its constructor.

This pattern of related interfaces, classes, and constructors enables code reuse and customization. Anticipating the need for customization, vendors can apply it in designing utility classes and frameworks, to facilitate their use in multiple inheritance contexts.

The pattern is appropriate in cases where the ability to customize inherited methods (for example, start ()) by overriding one or a few methods on which they rely (for example, run()) is required, but the ability to substitute child instances for parent instances (for example, Applets for Threads) is not relevant or impossible to implement.

But why didn't the designers of Java define a more complex interface than Runnable? It would have created the opportunity for conflicts. For instance, the start() method is defined in both classes - Applet and Thread - and with different semantics, while class Clock can support only one definition of start(). As explained in Section 3.1 such conflicts prohibit using Clocks as Threads. So a larger interface than Runnable would not have been beneficial anyway but would have added the risk of subtle errors.

\subsubsection{Marker interfaces}

In object-oriented languages that support multiple inheritance, general purpose facilities such as storing and retrieving objects from persistent storage, cloning objects, etc. can be defined as library classes and used as ancestors by classes needing such facilities. For instance, in Eiffel, the library class Storable defines I/O methods for objects: store(file:FILE) $\{\ldots\}$, Storable retrieved(file:FILE) $\{\ldots\}$, etc. In Java, such functionality can be achieved in many cases without taking recourse to multiple inheritance, as discussed below.

A marker interface is an interface that declares no methods or fields. It is useful when we need to determine something about the objects without assuming that they are instances of any particular class. (See [18] for examples.)

In Java, certain general purpose methods are housed in the root class java.lang.Object and made available only when a subclass implements a suitable marker interface. For instance, the clone()-method is defined in class java.lang.Object as protected and is inherited by all classes. However, invoking it on an arbitrary object can cause CloneNotSupportedException to be thrown. To run the clone()-method on an instance of a class, the class must implement the marker interface Cloneable and redefine clone(). 
Java classes such as java.io.ObjectInputStream and java.io.ObjectOutputStream support readObject (java.lang.Object) and writeObject(java.lang.Object), respectively, to read from/write to a persistent storage. Java also requires a class to implement the marker interface Serializable before it can participate in these methods. Observe that the signatures of these methods are "inverted" when compared with the corresponding methods of class Storable in Eiffel.

Cloning and storing objects are the only examples that cannot be regarded as instances of our translation. The cloning mechanism is hardwired in a native method of class Object; the serialization mechanism makes heavy use of reflection in order to determine whether the implementor of the Serializable interface provides its own private writeObject(java.io.ObjectOutputStream) method. In fact both make better examples of functionality that cannot be modeled in a way that is recommendable as an idiom.

\subsubsection{Static versus dynamic composition}

The lack of multiple inheritance does prohibit certain reasonable static combinations of built-in classes in Java. For instance, one cannot define a class of objects that can be used both as a java.io.LineNumberReader and as a java.io.InputStreamReader, or as a java.io. LineNumberReader and as a java.io.PushbackReader.

Instead, Java just provides constructors to turn any Reader instance into an instance of LineNumberReader, InputStreamReader or PushbackReader by applying the decorator pattern [19]. Replacing anticipated, static composition of different abstractions via multiple inheritance by demand-driven, dynamic composition via aggregation is a gain of functionality, not a loss.

\section{Additional language support}

So far we have seen that simulations of multiple inheritance perform very well on some examples and bad on others. This is not a really satisfactory conclusion. Can we do better? In this section we look at what can be gained by providing language support for the translations discussed above. We analyze two mechanisms: implementation of the implicit interface of a class and built-in delegation.

\section{1. similar}

In order to ease the implementation of scenarios which require substitutability of child instances for parent instances, let us first explore a new construct for class definition, called similar, with the following semantics:

class $C$ similar $D\{\ldots\}$ declares that class $C$ implements all the public methods in class D and that $\mathrm{C}$ instances may be used for $\mathrm{D}$ instances.

This construct permits omission of explicit interface declaration for D (which was required in the earlier translation). However, code reuse still entails defining a D-type field in class C, and forwarding some method calls to it while redefining others.

This approach can lead to name conflicts and is therefore subject to the complications discussed earlier. Compare, for instance

class Clock extends Applet similar Thread $\{\ldots\}$;

with the discussion of the Clock applet given earlier. 
So, to simplify matters, it is tempting to require that the superclass of $\mathrm{C}$ and the classes similar to $\mathrm{C}$ have disjoint methods. However, the semantics of forwarding captured by these translations may not be appropriate as explained below.

\subsection{Built-in delegation}

We now discuss an alternative proposal to simulating delegation in Java, as incorporated in Lava [20]. Lava extends Java with constructs for type-safe automatic forwarding. In particular, Lava supports two variants of forwarding: consultation and delegation. The following description of these variants has been adapted from [21].

An object, called the child, may have modifiable references to other objects, called its parents. Method calls for which the receiver has no matching method are automatically forwarded to its parents. When a suitable method is found in a parent (the method holder), it is executed after binding its implicit this parameter, which refers to the object on whose behalf the method is executed. Automatic forwarding with binding of this to the original method call receiver is called delegation, while automatic forwarding with binding of this to the method holder is called consultation. Delegation is object-based inheritance whereas consultation is just an automatic form of method calling.

Lava uses the keywords delegatee and consultee to mark instance fields of a child class that hold references to parent objects. These keywords specify whether to interpret forwarded method calls via delegation or via consultation, respectively. The two approaches differ when a parent method has been redefined in a child. The delegatee will invoke the redefined child method, while the consultee will invoke the original parent method. Alternatively, one can say that delegation supports overriding whereas consultation blocks it, as illustrated below.

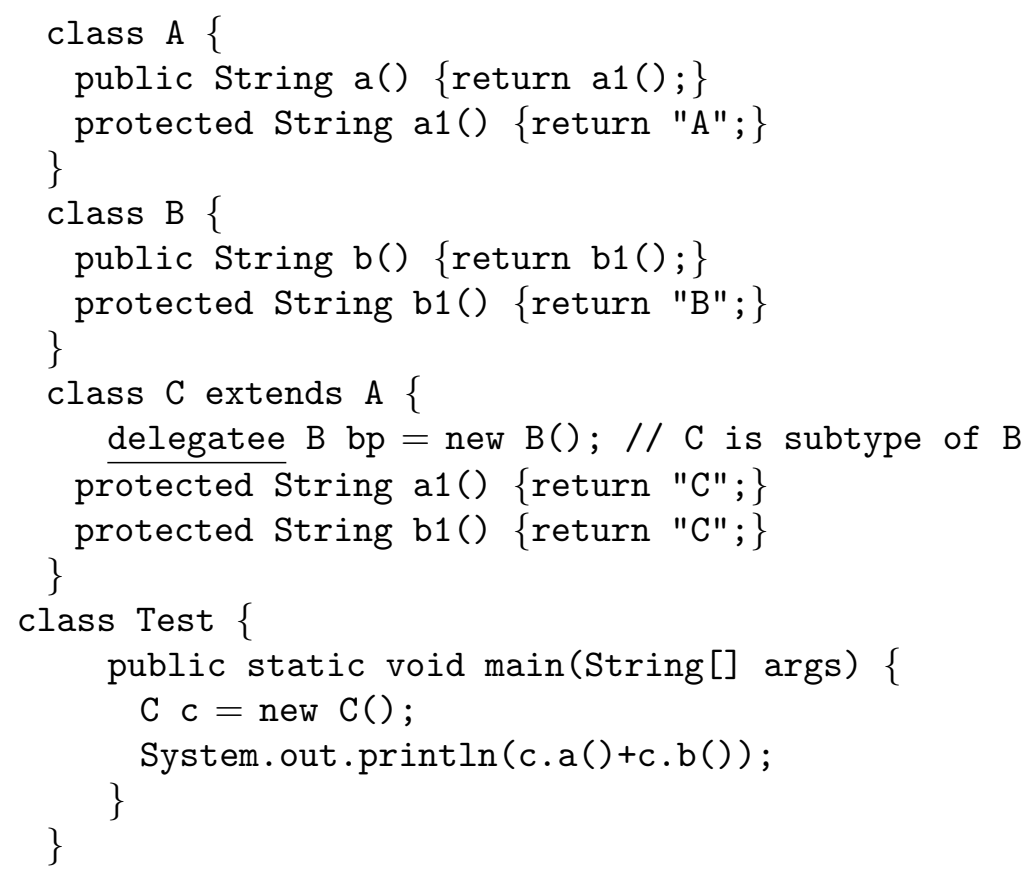


The expected outcome is "CC". If we used consultation the outcome would be "CB".

Note also that an object of class C can be treated as an object of both class A and class B. Delegation and consultation both have the effect of extending the interface of the child class by the public and protected methods of the declared parent types. It is instructive to compare the above code with the initial multiple inheritance example and the simulation of delegation.

\subsubsection{Conflict resolution}

In contrast with ordinary class-based languages, there are situations where conflicts can arise only at run-time. This can happen because, with delegation, the parent object may be any instance of the declared parent type or of one of its subtypes. In the latter case, it may contain additional methods, which may have the same signature as methods in the child object.

Such conflicts, that cannot be detected statically, are resolved in Lava by a "semantic compatibility" criterion that a method in a child may only override a method of a parent object if both override the same method in a common declared supertype of the child's class and the parent's class. Consider the following examples from [21].

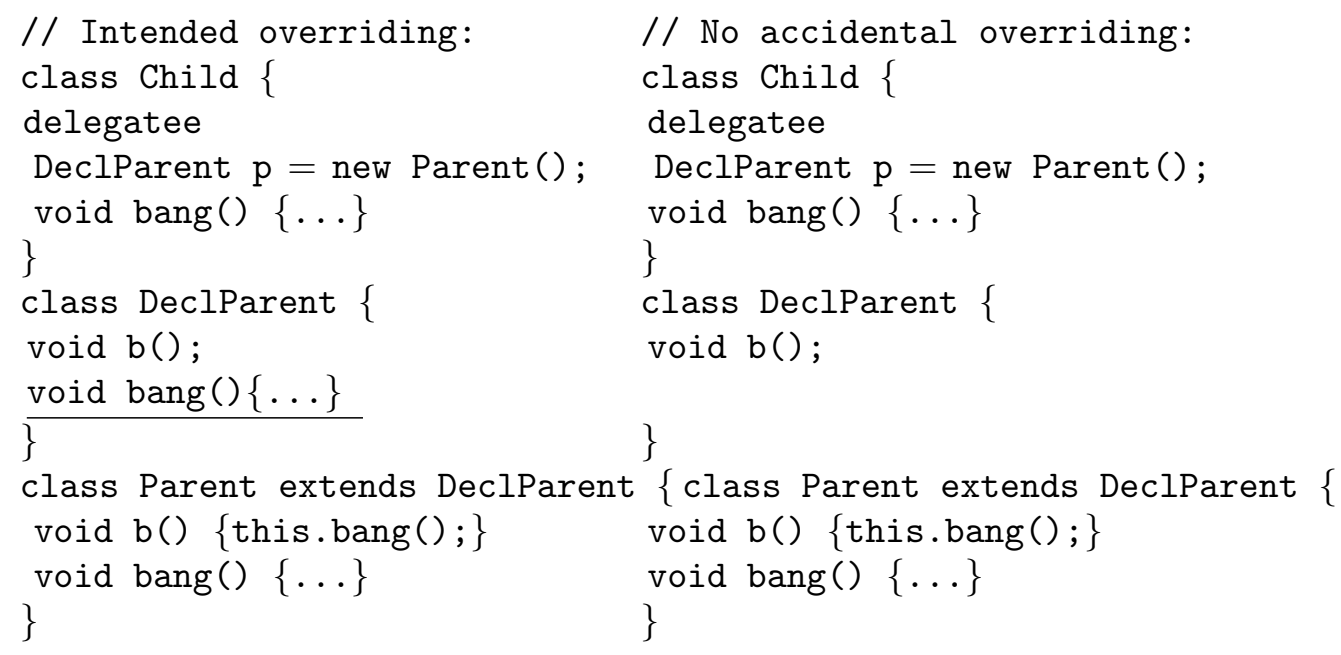

Let $c=$ new $\operatorname{Child}()$. Then $c . b()$ delegates $b()$ to $p$, which calls bang () on $c$ (recall that delegation has the effect of binding this to the object that initially received the forwarded message).

In Lava, for the left example, bang() in class Child will be invoked, thus overriding Parent's bang () - this is sensible to do because both methods are derived as specializations of the one in DeclParent - hence it can safely be assumed that they have a compatible semantics.

For the right example, the bang() method of class Child will not be selected because there is no evidence that it has the semantics expected in the calling context. Therefore, the bang () call will be forwarded to the parent object $\mathrm{p}$ - just like any other call that finds no applicable method in $\mathrm{c}$ - and the bang () method of $\mathrm{p}$ will be selected. The net effect is that accidental overriding is barred, preventing cumbersome and hard to locate errors. 


\subsubsection{Multiple delegation}

In [9], an extension of Lava is discussed in which a class can have multiple forwardees delegatees and consultants - that define methods under the same name. Such ambiguities have to be resolved, by explicit overriding. This construct is a simplification of Eiffel's redefine-renameundefine mechanism, as illustrated below. The notation

\section{localName(args) overrides field <-parentName}

means that the respective local method overrides the method parentName(args) in objects referenced by field. If the parentName is the same as localName it may be omitted, like in the following definition of clone() in class Child:

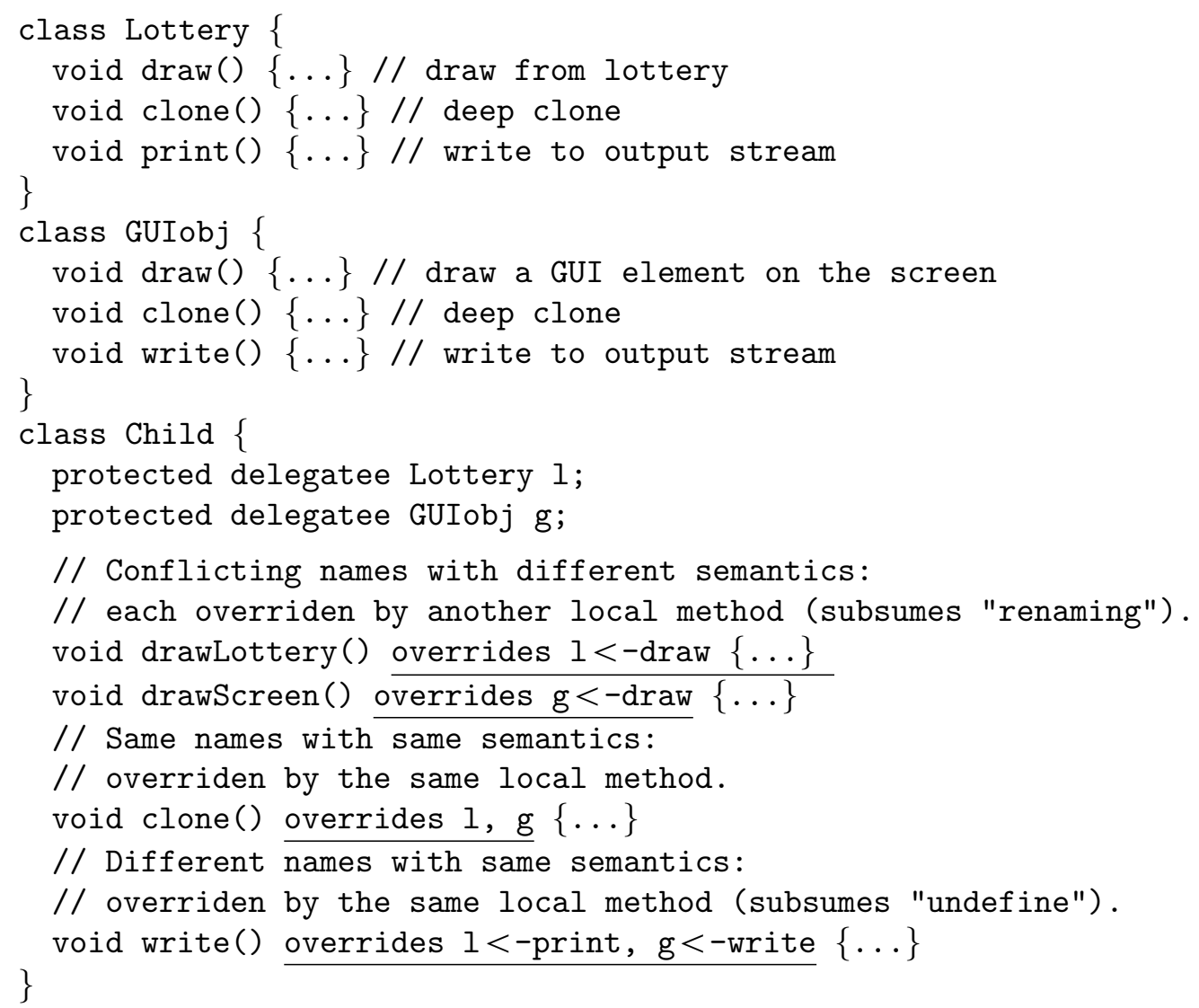

Multiple delegation also enables repeated delegation (analogous to repeated inheritance). Therefore, it raises the semantic problem pointed out for Eiffel's select statement in Section 3. A detailed analysis of this problem in the context of delegation, and the development of a solution are contained in [9]. A deeper discussion of explicit overriding can also be found there.

\subsection{Summary}

This section complemented the "pure Java" simulation by a discussion of possible language extensions that support forwarding-based designs. 
The discussion demonstrated that asking for multiple inheritance in Java is not a good idea given that more powerful, dynamic alternatives are available. If the language should ever be extended, support for (single or multiple) delegation would be preferrable to adding multiple inheritance. The primary advantage is the replacement of anticipated, static composition of classes via inheritance by unanticipated (mixin-style) dynamic composition of objects via delegation. This holds even for static delegation. Dynamic delegation offers the added benefit of being able to model dynamic evolution of object structure and behavior.

\section{Approximating generics in Java}

A class is an implementation of a data type. The "container" data types (such as the Set, the List, the Tree, etc.) can be abstracted and parameterized with respect to the type of the elements, in order to model homogeneous data structures (which contain just one type of elements). Such a parameterized type/module can be instantiated before use by binding concrete types to generic type parameters. For instance, one can instantiate List generic type/class to obtain various concrete types/classes such as List of integers, List of strings, List of dates, etc. These examples illustrate unconstrained genericity.

In contrast, in certain other situations, a generic type parameter may be partially constrained. For instance, a Sort routine or a Dictionary type may require the elements to be from a LinearOrder type. The templates in $\mathrm{C}++$, the generics in Eiffel and Ada-95, the functors in SML [22], etc., all support convenient implementation of such types (resp. sub-programs) as parameterized classes (resp. routines) where the type of a generic parameter expresses additional requirements. These parameterized modules are then suitably instantiated by binding types that satisfy the necessary constraints, to generic type parameters. This is known as constrained genericity.

Java does not support any construct akin to templates or generics. An extension to the Java language and the Java Virtual Machine is proposed in [23] to incorporate templates in their full glory. Pizza [24] and GJ [25] are supersets of Java which support parametric polymorphism that can be translated into pure Java. Here we review and analyze two idioms to approximate generic modules: one that uses the class Object and other reference types as the type of the generic element, and another that uses reflection. The first approach is exemplified by various utility classes of the Java 2 collections framework, while the second approach is illustrated using an example developed in Section 5.3. Additionally, a marker interface, as discussed in Section 3.2, can be used to express the requirement that the elements of a Dictionary must be linearly ordered.

\subsection{Using class Object}

A generic class parameter in a $\mathrm{C}++$ template can be straightforwardly translated into Java by turning it into a parameter of type Object, the root of the Java class hierarchy. However, this translation is only approximate since the Java class types are reference types [27] as explained below.

- A $\mathrm{C}++$ generic class parameter can be instantiated to a primitive type such as int, char, etc., as well as a class type, while, in Java, only the latter can be simulated. To instantiate a generic 
class parameter to a primitive type, Java requires the primitive type to be objectified using the corresponding wrapper class such as Integer, Character, Boolean, etc. Because these wrapper classes implement immutable objects, their use does not impact the semantics of assignment, the parameter passing mechanism, and the interpretation of final variables: for immutable objects these operations notice no difference between "copy semantics" vs. "reference semantics", "call by value" vs. "call by reference", and "immutable variable" vs. "immutable reference to immutable object". However, equality is not captured well by the translation: whereas $1==1$ is true, new Integer(1) == new Integer(1) is false. Here the difference between "value semantics" vs. "reference semantics" can only be concealed if we use the (value based) equals () method instead of "==". In Java, equals() is a method of class Object, and thus available on any object.

- Simulation of instantiation of a generic class parameter with a class type (as opposed to a primitive type) is also convoluted. For instance, to guarantee type safety, Java requires explicit type casts in expressions that use functions returning values of the generic parameter type. (Observe that, in the Java translation, such functions have a return type Object.) Consider the following template, which illustrates unconstrained genericity:

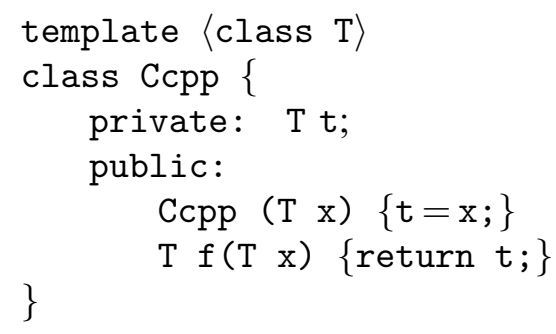

and a first approximation to it in Java:

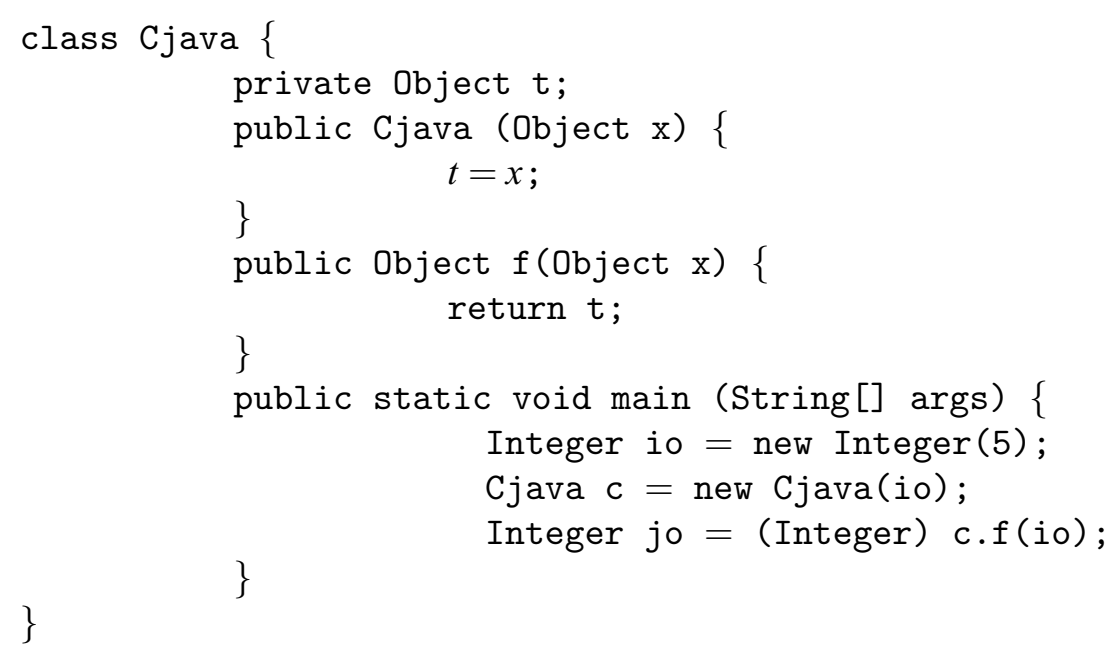

In the above Java translation, the method $f$ is not restricted to Integer argument. To impose the necessary type constraint, one can use inheritance and redefinition. However, to override a parent method in a subclass CIntjava, Java requires that the signature of the overriding method be the same as that of the corresponding parent method. (Otherwise, the new definition is treated as a valid 
overload.) So extra type checking code is needed to preserve the behavior. Observe also that an instance of CIntjava can be assigned to a variable of type Cjava.

Even though the subclass has "method-stubs" of the order of the number of methods in the parent class, it does not require duplication of method bodies.

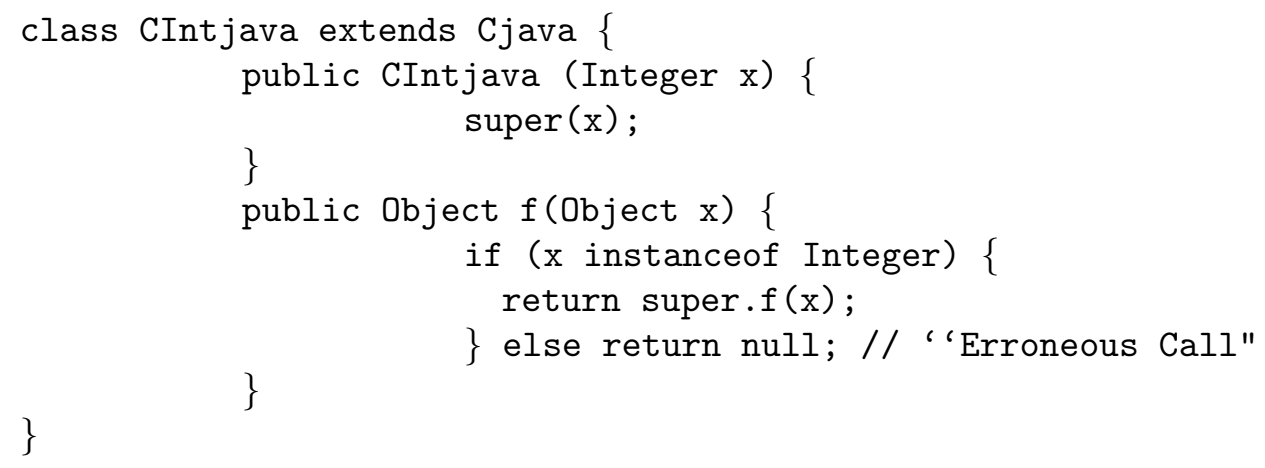

This approach resembles homogeneous translation of Pizza's polymorphism into Java [24].

Alternatively, one can change the signature of the method $f$ in CIntjava to accept and return only Integer objects. This can be accomplished using composition and forwarding. Unfortunately, all this can clutter up the code and introduce run-time overheads.

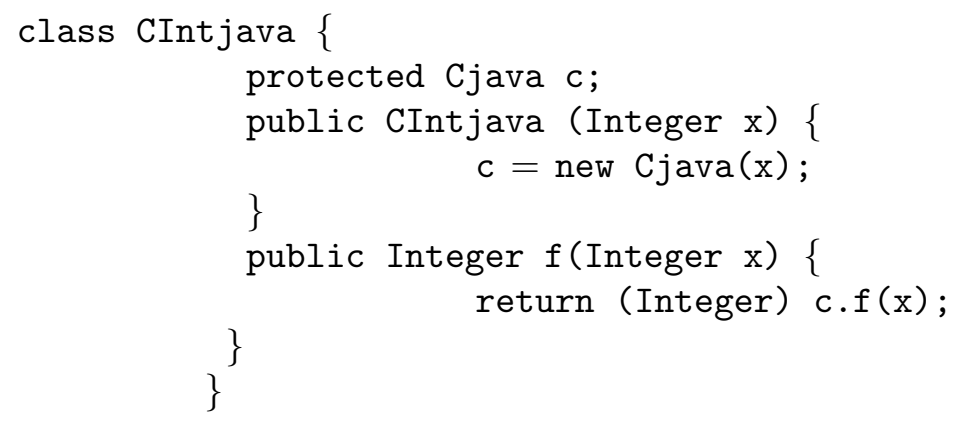

Observe that Java does not permit covariant typing of Eiffel [5] where a parent method can be overridden in the subclass using a method whose signature is more "restrictive" than the corresponding parent method. Observe also that Pizza's support for parameteric polymorphism improves readability, but has similar overheads due to translation into Java [24].

Refer to the Java utility classes/interfaces such as java.util.List, java.util.LinkedList, java.util.Hashtable, java.util.HashSet, etc for more examples.

\subsection{Using interface/class parameters}

Many parameterized modules require specification of additional semantic constraints or signature constraints on a generic parameter. The former corresponds to the expectations on the services provided by the actual argument and the latter on the requirements on the interfaces supported by the actual argument.

Languages such as SML, $\mathrm{C}++$, Eiffel, Ada-95, etc., all support such constrained genericity. In Java, such constraints can be expressed using class type method parameters to capture semantic 
constraints, and interface type method parameters to capture signature constraints in place of Object type parameters discussed earlier.

Refer to the uses of Java classes/interfaces such as java.awt.event.MouseListener, java. util.Iterator, java.util.SortedSet, java.util.TreeSet, java.awt.Container, etc., for additional examples.

The Java approach has one important limitation - certain type equality constraints cannot be expressed or enforced at compile time. For example, let $\mathrm{S}$ be a subclass of $\mathrm{C}$. Then, a collection of instances of C may potentially contain a mix of "direct" instances of C and "direct" instances of S. However, it is not possible to specify and verify statically (that is, without resorting to type tests and casts) a collection class that is made up entirely of either "direct" instances of C or "direct" instances of S, but not a mix of the two. See [26,24] for proposals to extend Java language to incorporate such constrained polymorphism.

The language GJ, which is a conservative extension of Java 2, supports generics including covariant overriding [25]. The translator for GJ into Java incorporates generalization of the examples sketched above.

\subsection{Using Java reflection}

As observed earlier, changes to a generic class (such as adding a method or a parameter to a method) can require explicit changes to all its "instantiation" subclasses. To minimize the impact of such changes, the Reflection API can be used. This is illustrated by the following Dictionary class example that is "parameterized" with respect to a linear order class as follows. (We have included the details for the interested readers; however, this example can be safely skipped. The Dictionary defined here is not to be confused with the obsolete abstract class java.util.Dictionary.)

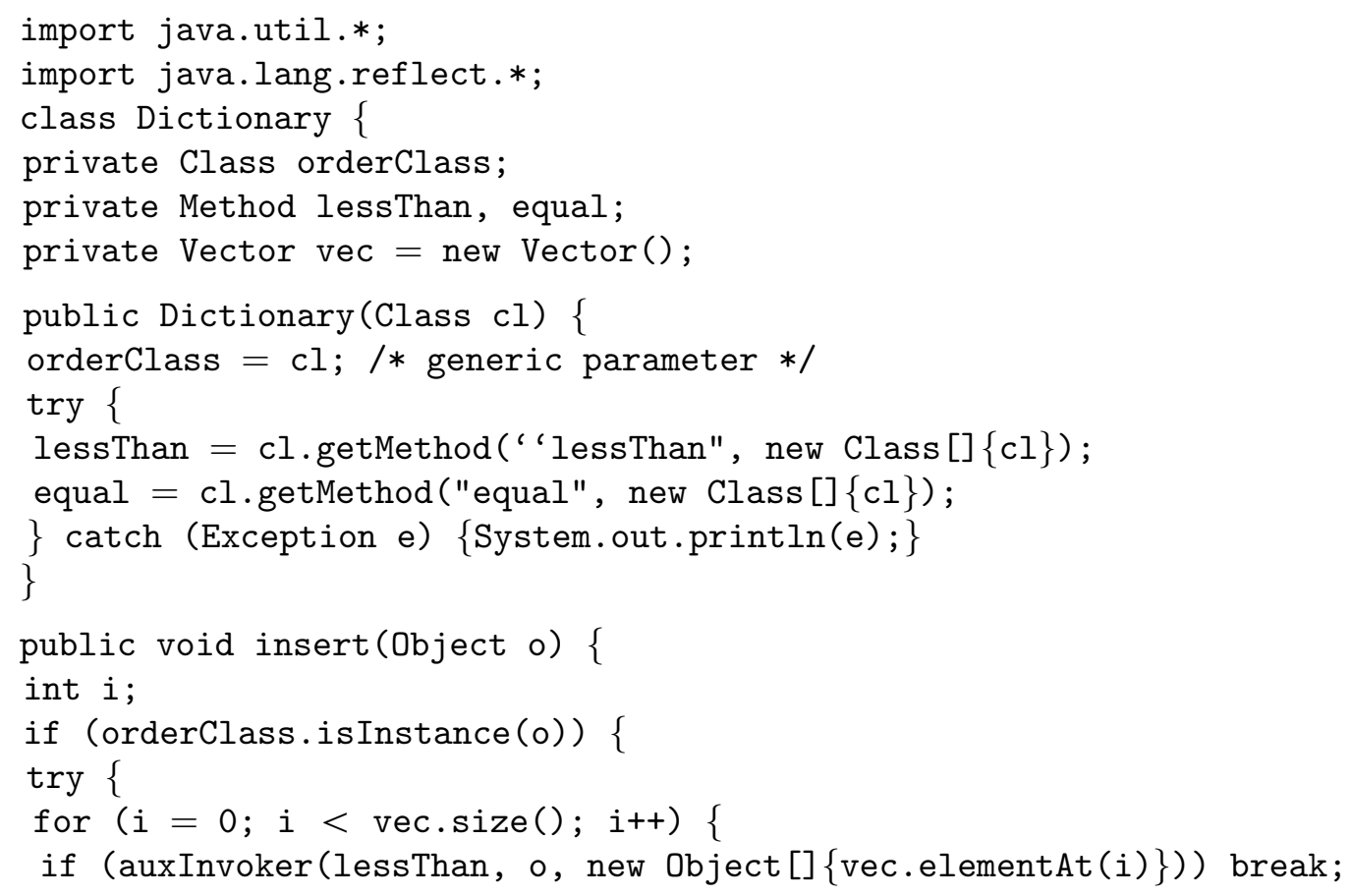




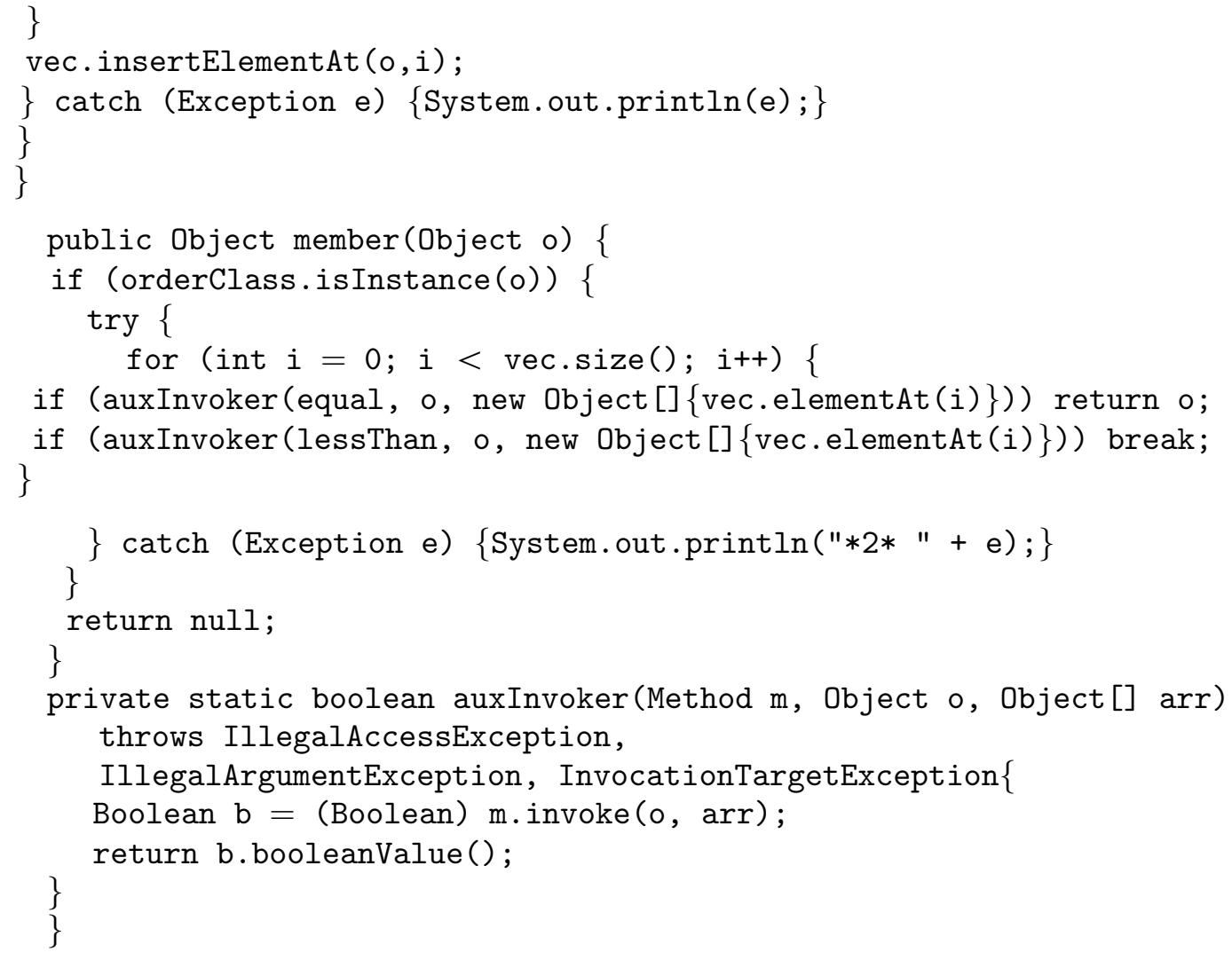

The above class can be used to implement a Dictionary of strings as follows.

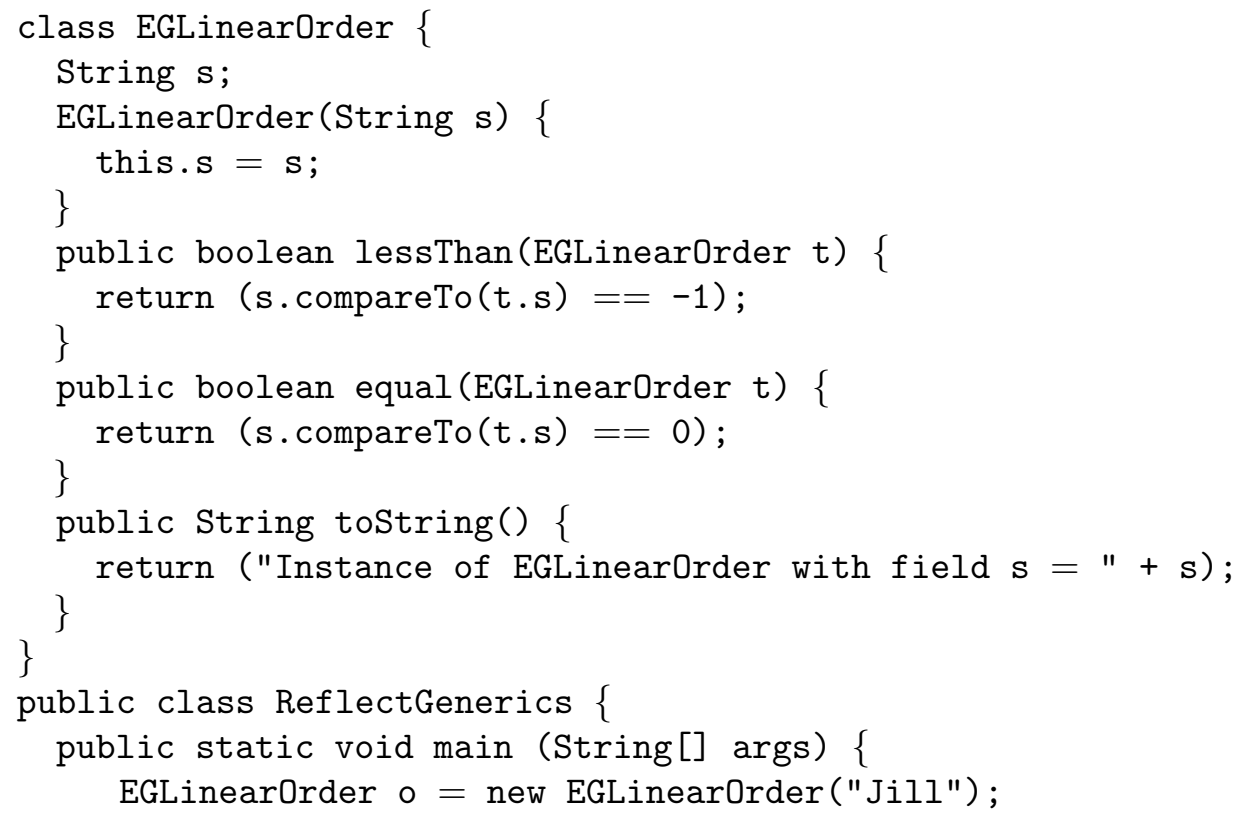




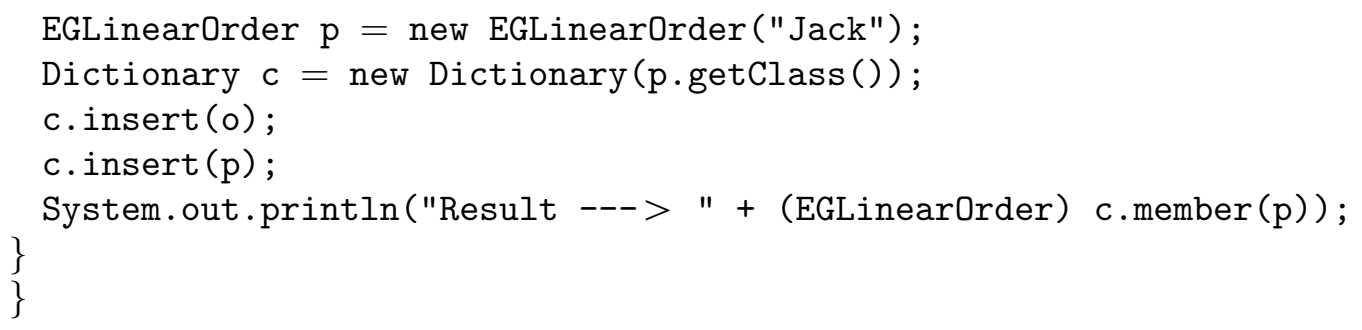

Even though the code for the generic Dictionary class using reflection looks complicated (in fact even downright "ugly"), the code for instantiation is straightforward. Unlike the non-reflective solution presented first, changes to the Dictionary class - such as adding a method public void delete(Object o) ... - do not require explicit changes to its instantiations. However, this approach cannot be used to instantiate generic parameters to primitive types, and will lose out on compile-time error checks. ${ }^{3}$ It is also clearly slower than the non-reflective simulation.

\section{Conclusions}

In this paper we reviewed Java idioms used by programmers to approximate generics and multiple inheritance of classes, to accrue reusability benefits.

Multiple inheritance was simulated by the joint use of forwarding as a means to achieve code reuse, interfaces as a means to achieve polymorphism, and back-references as a means to approximate overriding. The application of these techniques to various examples was demonstrated, shedding light on what can and cannot be achieved by the simulation.

An alternative proposal to simulating delegation in Java, as incorporated in Lava, which extends Java with constructs for type-safe automatic forwarding, was also discussed.

Finally, two approximations to generic modules were analyzed: one that uses the class Object and reference types as the type of the generic parameter (to capture unconstrained genericity and constrained genericity, respectively), and another that uses reflection. The former approach is not robust with respect to certain modifications to methods (such as adding a new method, or changing the signature of an existing method, etc.), while the latter approach leads to inefficient and difficult to read code.

\section{Acknowledgements}

We wish to thank the anonymous referees and Pascal Costanza for their comments and suggestions.

\footnotetext{
${ }^{3}$ Commercial software tools, such as PREfixco Corp.'s PREfix, that are based on software component simulation technology, may provide means to detect some errors prior to software deployment that would otherwise be caught only at run time.
} 


\section{References}

[1] Gamma E, Helm R, Johnson R, Vlissides J. Design patterns: elements of reusable object-oriented software. Reading, MA: Addison-Wesley, 1995.

[2] Buschmann F, Meunier R, Rohnert H, Sommerlad P, Stal M. Pattern-oriented software architecture: a system of patterns. New York: Wiley, 1996.

[3] Vlissides J. Pattern hatching. Reading, MA: Addison-Wesley, 1998.

[4] Joyner I. A C++?? critique. Eiffel Liberty Resources at http://www.elj.com/eiffel/ij//, 1998.

[5] Meyer B. Object-oriented software construction. 2nd ed. Englewood Cliffs, NJ: Prentice-Hall, 1997.

[6] Lakshmanan LVS, Thirunarayan K. Declarative frameworks for inheritance. In: Chomicki J, Saake G. editors. Logics for databases and information systems. Dordrecht: Kluwer Academic Publishers, 1998. p. 357.

[7] Stroustrup B. The C++ programming language. 3rd ed. Reading, MA: Addison-Wesley, 1997.

[8] Budd T. An introduction to object-oriented programming. 2nd ed. Reading, MA: Addison-Wesley, 1997.

[9] Kniesel G. Multiple inheritance and delegation revisited. University of Bonn, Germany, 2000, in preparation.

[10] Appel A. Modern compiler implementation in Java. Cambridge: Cambridge University Press, 1998.

[11] Driesen K, Hölzle U, Vitek J. Message dispatch on pipelined processors. In: Olthoff W. Proceedings ECOOP'95, Lecture Notes in Computer Science, vol. 952. Berlin: Springer, 1995. p. 253-82.

[12] Abadi M, Cardelli L. A theory of objects. Berlin: Springer, 1996.

[13] Cardelli L, Wegner P. On understanding types, data abstraction, and polymorphism ACM Computing Surveys 1985; 17(4):471-522.

[14] Harrison W, Ossher H, Tarr P. Using delegation for software and subject composition. Research Report RC 20946 (922722), IBM Research Division, T.J. Watson Research Center, 5 August 1997.

[15] Kniesel G. Delegation for Java - API or language extension? Technical Report IAI-TR-98-4, ISSN 0944-8535, University of Bonn, Germany, 1998.

[16] Blewitt A. Java tip 71: use dynamic messaging in Java. http://www.javaworld.com/javaworld/javatips/ jw-javatip71.html, 1999.

[17] Stroustrup B. The design and evolution of C++. Reading, MA: Addison-Wesley, 1994.

[18] Arnold K, Gosling J. The Java programming language, 2nd ed. Reading, MA: Addison-Wesley, 1999.

[19] Budd T. Understanding object-oriented programming with Java. updated ed. Reading, MA: Addison-Wesley, 2000.

[20] Kniesel G. Dynamic object-based inheritance with subtyping. Ph.D. thesis, University of Bonn, Computer Science Department III, 2000 .

[21] Kniesel G. Type-safe delegation for run-time component adaptation. European Conference on Object-Oriented Programming, Lecture Notes in Computer Science, vol. 1628. Berlin, Springer, 1999, p. 351-66.

[22] Ullman JD. Elements of ML programming. 2nd ed. (ML97). Englewood Cliffs, NJ: Prentice-Hall, 1998.

[23] Myers AC, Bank JA, Liskov B. Parameterized types in Java. Proceedings of 24th POPL, 1997. p. $132-45$.

[24] Odersky M, Wadler P. Pizza into Java: translating theory into practice. Proceedings of 24th POPL, 1997. p. 146-59.

[25] Bracha G, Odersky M, Stoutamire D, Wadler P. Making the future safe for the past: adding genericity to the Java programming language. Proceedings of OOPSLA-98, October 1998.

[26] Bruce K. Increasing Java's expressiveness with ThisType and match-bounded polymorphism. Draft 1997.

[27] Gosling J, Joy B, Steele G, Bracha G. The Java language specification. 2nd ed. Reading, MA: Addison-Wesley, 2000.

Krishnaprasad Thirunarayan received a Ph.D. in Computer Science from the State University of New York at Stony Brook in 1989, an M.E. in Computer Science from the Indian Institute of Science, Bangalore in 1984, and a B.Tech. in Electrical Engineering from the Indian Institute of Technology, Madras in 1982. He is currently an Associate Professor in the Department of Computer Science and Engineering at the Wright State University, Dayton, Ohio. His research interests are in Knowledge Representation and Reasoning, and Programming Languages: Design, Specification, and Implementation.

Günter Kniesel received a Ph.D. in Computer Science from the University of Bonn in 2000 and a Diploma (equivalent of an M.Sc.) in Computer Science from the University of Dortmund in 1989. He is currently a researcher in the Department 
of Computer Science at the University of Bonn, Germany. His current research interests are in object-oriented programming languages and design.

Haripriyan Hampapuram received a Ph.D. in Computer Science from the Rutgers University, New Jersey in 1994, an M.Sc. in Computer Science from the Indian Institute of Science, Bangalore in 1986, and a B.E. in Electronics from the University Visweswaraiah Engineering College, Bangalore in 1982. He has worked on products relating to software engineering and compilers at various companies including AT\& T Bell Laboratories, Philips Semiconductors, Intrinsa Corporation (now acquired by Microsoft Corporation). He is currently at Microsoft Research, Seattle, Washington developing a static analysis tool for finding defects in $\mathrm{C}$ and $\mathrm{C}++$ programs. 